\title{
Crossover from 3D Ising to Isotropic Lifshitz Critical Behavior in a Mixture of a Homopolymer Blend and Diblock Copolymer
}

\author{
Dietmar Schwahn, ${ }^{1}$ Kell Mortensen, ${ }^{2}$ Henrich Frielinghaus, ${ }^{1,2}$ and Kristoffer Almdal ${ }^{2}$ \\ ${ }^{1}$ Institut für Festkörperforschung, Forschungszentrum Jülich GmbH, D-52425 Jülich, Germany \\ ${ }^{2}$ Condensed Matter Physics and Chemistry Department, Ris $\phi$ National Laboratory, DK-4000 Roskilde, Denmark
}

(Received 5 November 1998)

Thermal composition fluctuations and the associated crossover from the $3 \mathrm{D}$ Ising to the isotropic Lifshitz universality class have been studied in a three component mixture made of a critical polymer blend and the corresponding diblock copolymer. The critical exponents were found to be appreciably larger than those of the 3D Ising, in agreement with expectations from the larger upper critical dimension. Very near the critical temperature a crossover to a renormalized Lifshitz critical behavior was observed possibly caused by fluctuation induced rearrangements of the diblock copolymers. [S0031-9007(99)09485-5]

PACS numbers: 61.25.Hq, 64.60.Cn, 64.60.Fr

Studies of thermal composition fluctuations in binary mixtures of homopolymers and diblock copolymers have produced a good understanding of the critical behavior [13]. The critical exponents are of the mean field universality class far from the critical point, whereas fluctuations affect the exponents in the near vicinity of the critical point. The crossover temperature from mean field to nonmean field is estimated by the Ginzburg criterion [1,2], which predicts a $1 / N$ and $1 / \sqrt{N}$ ( $N$ degree of polymerization) scaling behavior for blends and diblock copolymers, respectively. In binary polymer blends the 3D Ising critical range has been observed to be appreciably larger than that estimated from the $1 / N$ scaling law [3], an observation which is mainly attributed to the effect of compressibility [4]. In diblock copolymers the deviation from mean field behavior is even larger and the critical point is replaced by a first order phase transition [5]. Fluctuations in the more complex mixture of a critical binary homopolymer blend and the corresponding symmetrical diblock copolymer lead to the universality class of the isotropic Lifshitz critical behavior as the order parameter is a scalar $(n=1)$ and the dimension in which the wave vector instability occurs $(m)$ is equal to the spatial dimension $(d), m=d=3$ [6-11]. In such systems one might expect significant renormalization due to thermal fluctuations as the upper critical dimension is 2 times larger $\left(d_{U}=8\right)$ than that of binary blends $\left(d_{U}=4\right)$ [9]. This also makes it difficult to calculate the critical exponents [8]. In a recent study on such a polymer mixture mean field critical behavior was surprisingly observed even rather near the isotropic Lifshitz critical point [11]. In the present Letter we report equivalent experiments, however, with a mixture of significantly reduced polymer masses. This study demonstrates a transition from mean field to $3 D$ Ising behavior at small copolymer contents, and a transition from mean field to isotropic Lifshitz critical behavior at higher copolymer contents. The observed critical exponents near the Lifshitz point are significantly larger than those of the 3D Ising universality class.
The principal effect of diblock copolymers solved in a homopolymer blend is the reduction of the surface energy which according to the corresponding Hamiltonian of the

$$
\begin{gathered}
H=\frac{1}{2} \int d^{d} x\left\{c_{2}(\nabla \varphi)^{2}+c_{4}\left(\nabla^{2} \varphi\right)^{2}+r \varphi^{2}\right. \\
\left.+u \varphi^{4}+u_{6} \varphi^{6}\right\}
\end{gathered}
$$

order parameter $\varphi$ is described by a reduction of the parameter $c_{2}$. This $c_{2}$ parameter is positive at low copolymer content, becomes zero at the composition of the Lifshitz critical point (Lifshitz line), and is negative for large copolymer content. Near the Lifshitz line the higher order term $c_{4}\left(\nabla^{2} \varphi\right)^{2}$ of the gradient energy therefore becomes important. For a positive $c_{2}$ parameter, one has the characteristic behavior of polymer blends with $r \equiv S^{-1}(0)=0$ at the critical temperature. The inverse susceptibility $S(0)$ is identical to the forward scattering $S(Q=0)$ ( $Q$ momentum transfer) measured in scattering experiments. At the Lifshitz critical point one has the two conditions $S^{-1}(0)=0$ and $c_{2}=0$. The decrease of the surface energy leads to a structure factor with an additional $Q^{4}$ term, whose coefficient $B$ is

$$
\begin{aligned}
S^{-1}(Q) & =S^{-1}(0)+A Q^{2}+B Q^{4} \\
& =S^{-1}(0)\left[1+(Q \xi)^{2}+p^{-2}(Q \xi)^{4}\right],
\end{aligned}
$$

proportional to the $c_{4}$ term in the Hamiltonian. The second expression of Eq. (2) defines the correlation length $\xi=\sqrt{S(0) A}$ of the thermal fluctuations and the gradient term $p^{-2}=B / A^{2} S(0)$ [8]. At the Lifshitz critical temperature the correlation length and the parameter $p$ become constant $(A=0)$; in this case $\xi$ loses its meaning and has to be redefined from the $Q^{4}$ term. At smaller copolymer content, $\xi$ and $p$ become infinite at the critical temperature according to the scaling law $p^{2} \propto \xi^{(2+\eta)} / B$ with $A \propto \xi^{\eta}$ [12] and the Fisher exponent $\eta=2-\gamma / \nu$. The parameters $\gamma$ and $\nu$ are the critical exponents of the susceptibility and correlation length, respectively. 


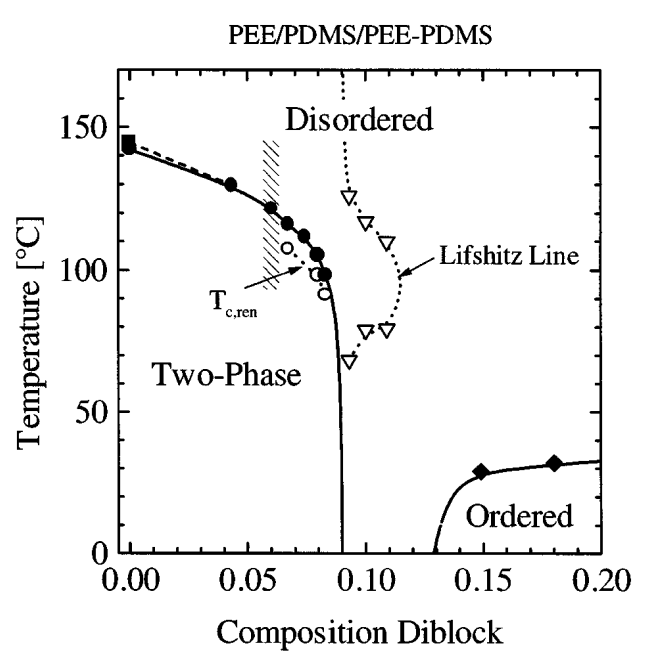

FIG. 1. Phase diagram. The filled circles represent the critical temperatures of the 3D Ising and isotropic Lifshitz case separated by the dashed area, the open circles the critical temperatures of the renormalized Lifshitz, the square the binodal, the diamonds the order-disorder transition, and the triangles the Lifshitz line.

Small-angle neutron scattering was used to measure thermal composition fluctuations in the ternary mixture of poly(ethyl ethylene), PEE, and poly(dimethylsiloxane), PDMS, and a number of different compositions between $\phi=4.3 \%$ and $\phi=8.3 \%$ volume fraction of the corresponding symmetric diblock copolymer, PEE-PDMS. The polymers were all synthesized by anionic polymerization followed by catalytic hydrogenation [13]. The PEE monomers were partly deuterated during a catalytic saturation as only a limited isotope exchange takes place during such a process [14]. Based on the measured densities, the chemical formula of the PEE monomers are $\mathrm{C}_{4} \mathrm{D}_{2.8} \mathrm{H}_{5.2}$. The scattering contrast between PEE and PDMS leads therefore to the observation of thermal fluctuations of the total monomer fraction. As we study a symmetric ternary mixture with equal concentrations of PEE and PDMS monomers the Hamiltonian in Eq. (1) describes the system near the consolute line [8]. The degree of polymerization was $N_{\text {PEE }}=30.5, N_{\text {PDMS }}=29.2$, and $N_{\text {PEE-PDMS }}=$ 168 leading to the ratio $\alpha=\sqrt{N_{\mathrm{PEE}} N_{\mathrm{PDMS}}} / N_{\mathrm{PEE}-\mathrm{PDMS}}=$ 0.18 [7]. The homopolymers were always mixed with a PEE volume fraction of 0.516 . The scattering experiments were performed at the KWS1 small angle neutron scattering (SANS) diffractometer at the FRJ-2 research reactor of the FZ-Jülich [15]. The composition fluctuations were measured in situ at the corresponding temperatures. The scattered neutron intensity was corrected from background contributions and calibrated in absolute units by a Lupolen secondary standard.

The phase diagram of the ternary PEE/PDMS/PEEPDMS system with copolymer content $\Phi$ is shown in the range of $\Phi=0 \%-20 \%$ in Fig. 1. A phase diagram including the whole $\Phi=0 \%-100 \%$ will be published else-

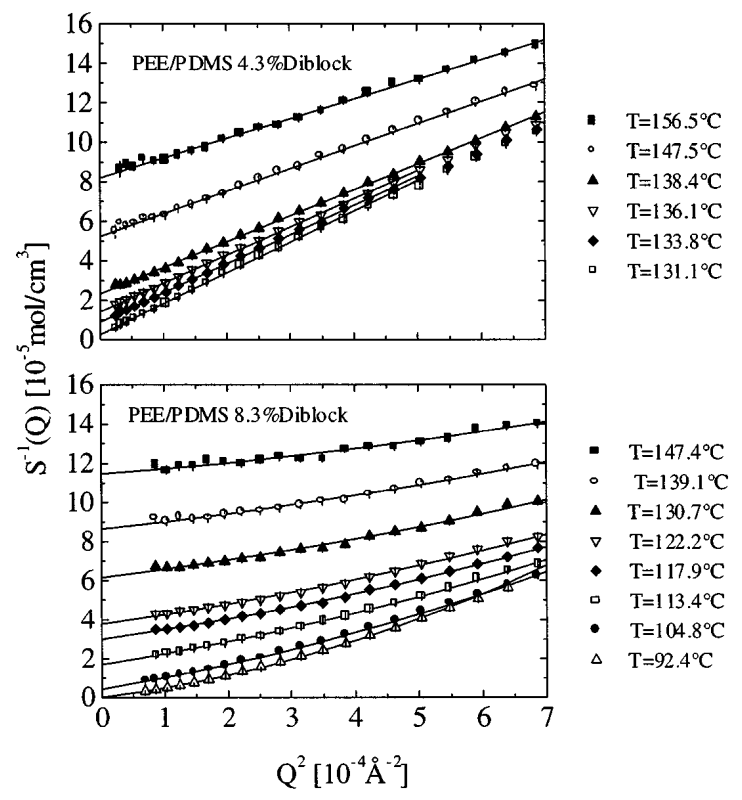

FIG. 2. Structure factor in Zimm representation.

where [16]. The disordered regime at high temperatures is separated by the Lifshitz line. At diblock composition lower than the Lifshitz line, the maximum intensity occurs at $Q=0$ while for $\Phi$ larger than the Lifshitz line the maximum intensity is observed at a finite $Q^{*}$ value. Theoretically, the Lifshitz line is estimated with $6 \%$ according to $\Phi_{\mathrm{LP}}=2 \alpha^{2} /\left(1+2 \alpha^{2}\right)$ [6]. The open triangles (dashed line guide for the eye) of the Lifshitz line are experimental points [17]. They depend on composition in contrast to theoretical predictions; its smallest composition is found at $9.3 \%$ and its largest value around $100{ }^{\circ} \mathrm{C}$ at $10.9 \%$. At low and high temperatures the Lifshitz line appears constant at

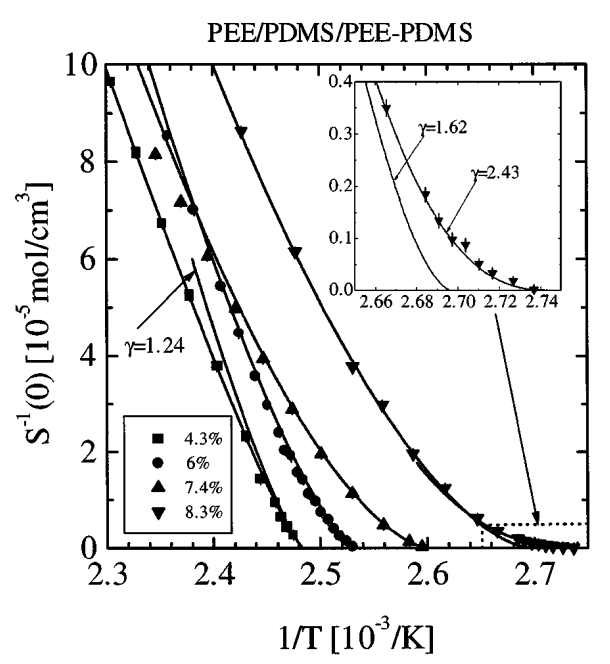

FIG. 3. Inverse susceptibility versus inverse temperature. $S(0)$ in the $4.3 \%$ sample follows a crossover function between mean field and 3D Ising case while $S(0)$ of the other samples follows a scaling law with larger critical exponents. Inset shows $S(0)$ of the $8.3 \%$ sample near $T_{c}$. 
TABLE I. Parameters of the isotropic Lifshitz case. The Ginzburg numbers indicated with $(*)$ were estimated from $T_{c} / T_{c}(\mathrm{MF})=$ $(1-\mathrm{Gi}) ; T_{c}(\mathrm{MF})$ is the critical mean field temperature [3].

\begin{tabular}{lccccccc}
\hline \hline$\Phi(\%)$ & $T_{c}\left({ }^{\circ} \mathrm{C}\right)$ & $\mathrm{Gi}\left(10^{-2}\right)$ & $C_{+}\left(\mathrm{cm}^{3} / \mathrm{mol}\right)$ & $\gamma$ & $\xi_{0}(\AA)$ & $\nu$ & $\eta=2-\gamma / \nu$ \\
\hline 4.3 & 129.7 & $1.5 \pm 0.6\left(\cong 0.7^{*}\right)$ & $323 \pm 148$ & 1.24 & $\ldots$ & 0.632 & 0.038 \\
6 & 121.9 & $\cong 1.8^{*}$ & $293 \pm 60$ & $1.37 \pm 0.03$ & $3.5 \pm 0.3$ & $0.84 \pm 0.01$ & $0.37 \pm 0.01$ \\
6.7 & 116 & $\cong 1.9^{*}$ & $227 \pm 30$ & $1.62 \pm 0.02$ & $2.7 \pm 0.6$ & $0.95 \pm 0.03$ & $0.29 \pm 0.01$ \\
7.4 & 111.4 & $\cong 2.6^{*}$ & $269 \pm 34$ & $1.61 \pm 0.02$ & $2.2 \pm 0.5$ & $1 \pm 0.03$ & $0.39 \pm 0.01$ \\
8.3 & 97.9 & $\cong 4.3^{*}$ & $279 \pm 63$ & $1.62 \pm 0.03$ & $1.9 \pm 0.6$ & $1.03 \pm 0.05$ & $0.43 \pm 0.02$ \\
\hline \hline
\end{tabular}

about 9\%. Above the Lifshitz line mesoscopic ordering is expected to occur below an ordering temperature. Such an ordering, however, could be observed only for copolymer contents of $\Phi=15 \%$ and larger. Below $\Phi=15 \%$ experiments were performed down to $-10{ }^{\circ} \mathrm{C}$ without any ordering transition [17]. At compositions below the Lifshitz line ordering occurs on a macroscopic scale by phase separation. As the samples are mainly at critical composition the binodal and spinodal phase boundaries represent the consolute line depicted in the phase diagram by the open and closed dots (solid and dashed lines are guides for the eye). Only for $\Phi=0$ the system is slightly off critical composition. The Lifshitz critical point is a multiple critical point at the connection of the macroscopic and microscopic ordering transition lines. It can, however, be realized only within mean field approximation; according to the Ginzburg criterion thermal fluctuations differently stabilize the disordered phases in blends and diblock copolymers and thereby destroy the Lifshitz point [6-9]. This becomes visible by the gap of disorder between approximately $9 \%$ and $15 \%$ (Fig. 1) similar to observations in Ref. [11].

We limit our further discussions to the behavior of thermal composition fluctuations in the disordered regime below the Lifshitz line. The corresponding structure factors $S(Q)$ have been plotted for various temperatures in Fig. 2 in a Zimm representation: $S^{-1}$ vs $Q^{2}$. The solid lines represent fits according to Eq. (2) from which the susceptibility $S(0)$ and the two coefficients $A$ and $B$ emerge. In the $\Phi=4.3 \%$ sample, $S(Q)$ is sufficiently well described by the Ornstein-Zernike approximation corresponding to $B=0$. For larger block copolymer contents the $Q^{4}$ term becomes important. The inverse susceptibility $S^{-1}(0)$ vs $1 / T$ is shown in Fig. 3. The critical temperatures obtained at $S^{-1}(0)=0$ decrease with composition as depicted by the solid points in the phase diagram. In the $\Phi=4.3 \%$ mixture, $S^{-1}(0)$ behaves similarly to blends; namely, from high to low temperatures a transition from mean field to 3D Ising critical behavior is observed. This is demonstrated by the corresponding fit (solid line) of the crossover function by Belyakov et al. [18]. This crossover function has extensively been used by us for polymer blends $[3,4]$. We will not discuss its analytical form here, but only present the resulting parameters in Table I. A second line through some of the $4.3 \%$ data represents the asymptotic 3D Ising scaling law with the critical expo- nent $\gamma=1.24$ as evaluated from the crossover function. The inverse susceptibilities of the compositions $\Phi=6 \%$, $7.4 \%$, and $8.3 \%$ are characterized by curvatures far beyond that of the $\Phi=4.3 \%$. Such larger curvature corresponds to an increase of the $\gamma$ exponent, indicating a crossover to the universality class of the isotropic Lifshitz case. These susceptibilities could be analyzed by the scaling law according to $S^{-1}(0)=C_{+}^{-1} t^{\gamma}$ with the reduced temperature, $t=\left(T-T_{c}\right) / T$, and the critical exponent, $\gamma$, as demonstrated by the solid lines. A similar analysis has been performed for the correlation length according to $\xi=\xi_{0+} t^{-\nu}$ with the critical exponent $\nu$. The obtained critical exponents are collected in Table I and are shown versus the copolymer content in Fig. 4. The corresponding $T_{c}$ are depicted as full dots in the phase diagram of Fig. 1. In two samples with composition 6.7\% (not shown in Fig. 3 for clarity) and $8.3 \%$ an additional crossover was observed very near the critical temperature and phase separation was observed at a lower critical temperature plotted as open dots in the phase diagram. In this range the susceptibilities
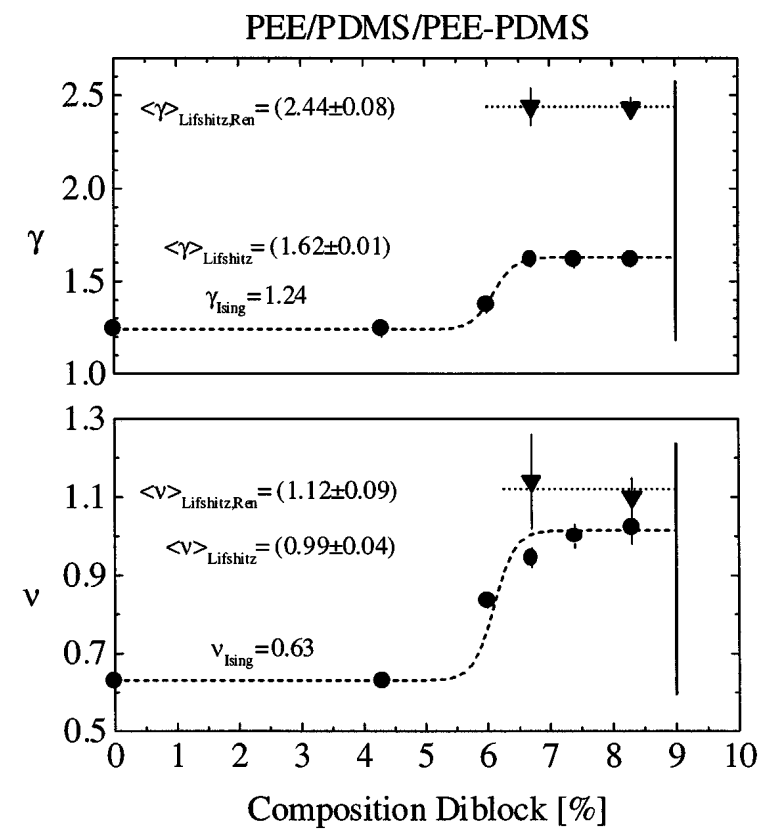

FIG. 4. Critical exponents of susceptibility and correlation length. At about $6 \%$ diblock content a crossover from 3D Ising to isotropic Lifshitz critical behavior is observed. Near $T_{c}$ a further crossover to a renormalized Lifshitz critical behavior was observed. 
TABLE II. Parameters of the renormalized isotropic Lifshitz case.

\begin{tabular}{ccccccc}
\hline \hline$\Phi(\%)$ & $T_{c}\left({ }^{\circ} \mathrm{C}\right)$ & $C_{+}\left(\mathrm{cm}^{3} / \mathrm{mol}\right)$ & $\gamma$ & $\xi_{0}(\AA)$ & $\nu$ & $\eta=2-\gamma / \nu$ \\
\hline 6.7 & 107.4 & 78 & $2.44 \pm 0.1$ & 3.5 & $1.14 \pm 0.12$ & $-(0.14 \pm 0.02)$ \\
8.3 & 91.4 & 55 & $2.43 \pm 0.06$ & 2.4 & $1.1 \pm 0.05$ & $-(0.21 \pm 0.01)$ \\
\hline \hline
\end{tabular}

were analyzed by the same scaling law as demonstrated for the $8.3 \%$ sample in the inset of Fig. 3; the corresponding appreciably larger exponents and the critical temperatures are summarized in Table II and plotted in Fig. 4. On the other hand, in the $7.4 \%$ sample phase separation occurred at the higher critical temperature shown as a full dot in the phase diagram and no additional crossover was observed. This experiment was performed with larger temperature steps and therefore the sample spent much less time in the region of strong thermal fluctuations. The experimental procedure was always the same; after the sample reached the desired temperature within the limits of less than $0.1 \mathrm{~K}$ two experimental runs of 10 and 30 min were started. Based on these data we conclude that for the samples between $6 \%$ and $9 \%$ diblock content two consolute lines can be realized experimentally. These are plotted by the open and full dots in the phase diagram, respectively; phase separation occurs either at the upper or the lower lines, depending on whether the crossover to the new universality class can occur or not. Such a crossover, however, is observed only if the experiments are performed sufficiently slowly. We abbreviate this as the renormalized Lifshitz critical behavior. An interpretation of this crossover might be related to rearrangements of the diblock copolymers as caused by the strong thermal fluctuations near $T_{c}$ and which further stabilize the sample against phase decomposition.

To summarize, the numerical numbers of the critical exponents of the susceptibility and correlation length in Fig. 4 show the following: For compositions with block copolymer content below $5.5 \%$ the thermal fluctuations are described by the universality class of the 3D Ising behavior $(\gamma=1.24)$. Between $\Phi=5 \%$ and 7\%, approximately, the system crosses gradually over to the isotropic Lifshitz critical behavior characterized by larger critical exponents $(\gamma=1.62)$. Even larger critical exponents were obtained after the crossover to the Lifshitz renormalized state very close to the critical temperature $(\gamma=2.44)$. The critical exponents $\gamma$ and $\nu$ are related by the Fisher scaling relation to the Fisher exponent $\eta$ presented in Tables I and II. The experimental $\eta$ values are of the order of one magnitude larger than those of polymer blends in the 3D Ising regime, and become negative in the renormalized state. A crossover from 3D Ising to a Lifshitz and renormalized Lifshitz critical behavior is observed even though the Lifshitz critical point itself cannot be reached experimentally; it is suppressed by strong thermal composition fluctuations.

We thank M. Heiderich from FZ-Jülich for technical support and Professor F.S. Bates and Professor T.P. Lodge from University of Minnesota for illuminating discussions.

[1] P.G. deGennes, Scaling Concepts in Polymer Physics (Cornell University Press, Ithaca, 1979).

[2] F. S. Bates and G. H. Fredrickson, Annu. Rev. Phys. Chem. 41, 525 (1990).

[3] D. Schwahn, G. Meier, K. Mortensen, and S. Janssen, J. Phys. II (France) 4, 837 (1994).

[4] D. Schwahn, T. Schmackers, and K. Mortensen, Phys. Rev. E 52, R1288 (1995).

[5] F. S. Bates, M. F. Schulz, A. K. Khandpur, S. Förster, J. H. Rosdale, K. Almdal, and K. Mortensen, Faraday Discuss. Chem. Soc. 98, 7 (1994).

[6] D. Broseta and G. H. Fredrickson, J. Chem. Phys. 93, 2927 (1990).

[7] G. H. Fredrickson and F. S. Bates, J. Polym. Sci. B, Polym. Phys. 35, 2775 (1997).

[8] R. Holyst and M. Schick, J. Chem. Phys. 96, 7728 (1992).

[9] L. Kielhorn and M. Muthukumar, J. Chem. Phys. 107, 5588 (1997).

[10] F. S. Bates, W. Maurer, T. P. Lodge, M. F. Schulz, M. W. Matsen, K. Almdal, and K. Mortensen, Phys. Rev. Lett. 75, 4429 (1995).

[11] F. S. Bates, W. W. Maurer, P. M. Lipic, M. A. Hillmyer, K. Almdal, K. Mortensen, G. H. Fredrickson, and T.P. Lodge, Phys. Rev. Lett. 79, 849 (1997).

[12] S. Janßen, D. Schwahn, and T. Springer, Phys. Rev. Lett. 68, 3180 (1992).

[13] K. Almdal, K. Mortensen, A. J. Ryan, and F. S. Bates, Macromolecules 29, 5940 (1996).

[14] N. P. Balsara, L. J. Fetters, N. Hadjichristidis, D. J. Lohse, C.C. Han, W.W. Graessley, and R. Krishnamoorti, Macromolecules 25, 6137 (1992).

[15] Neutronenstreuexperimente am FRJ-2 in Jülich (available at Forschungzentrum Jülich, 1997).

[16] M. A. Hillmyer et al. (to be published).

[17] Full manuscript is in preparation.

[18] M. Y. Belyakov and S. B. Kiselev, Physica (Amsterdam) 190A, 75 (1992). 